\title{
Bases para la conservación de las semillas de Calophyllum BRASILIENSE (CALOPHYLLACEAE)
}

\author{
CLAUDIA B. SOROL ${ }^{1}$, SOFÍA CARVAJAL², VERÓNICA CALONGA SOLÍS ${ }^{3}$, NAIKÉ LUCÍA \\ GONZÁLEZ ${ }^{4}$ y FABIANA ECKERS ${ }^{5}$
}

\begin{abstract}
Resumen: En Argentina la especie arbórea Calophyllum brasiliense Cambess (Calophyllaceae) constituye poblaciones en los bordes de esteros y en las selvas higrófilas del río Paraná. Las poblaciones de la margen del río forman parte de ecosistemas fuertemente fragmentados por su asociación a las cotas de inundación del embalse Yacyretá, lo cual las ha llevado a reducir drásticamente su tamaño al punto de encontrarse en riesgo de extinguirse localmente. Uno de los efectos de la fragmentación en las especies vegetales es la producción de semillas en menor cantidad, calidad o ambas. El valor ecológico, medicinal, económico y social de la especie y el estado de sus poblaciones suscitaron interés en conservarla. Una de las estrategias implementadas fue el almacenamiento de sus semillas, las cuales son recalcitrantes. Los objetivos del presente trabajo fueron evaluar tres poblaciones en cuanto a su potencialidad de producir semillas capaces de generar individuos y evaluar la calidad fisiológica en relación al momento de cosecha y al tiempo de almacenamiento bajo cuatro modos de almacenamiento. La población de Puerto Valle produjo semillas de buena calidad que conservaron la viabilidad durante treinta y un semanas cuando se almacenaron con endocarpo, en frascos de vidrio.
\end{abstract}

Palabras clave: Calophyllum brasiliense, semillas, almacenamiento, conservación, calidad fisiológica.

\begin{abstract}
Summary: Basis for conservation seeds Calophyllum brasiliense (Calophyllaceae). Calophyllum brasiliense Cambess (Calophyllaceae) is a tree species distributed in Argentina in the edges of matting and hygrophilous forests of the Paraná River. Those of the riverbank have been strongly fragmented due to the increment of the flood levels caused by the Yacyreta dam, thus, this populations are at risk of being locally extinguished as a consequence of their drastic size reduction. Fragmentation can affect plant species reducing the amount or the quality of seeds produced or both. The ecological, medicinal, economic and social value of the species and the status of their populations have generated interest in its preservation. One of the strategies implemented was the storage of its seeds, which are recalcitrant. The aims of this study were to evaluate three populations with respect to their potentiality to produce seeds capable of generating individuals, and evaluate the physiological quality regarding the harvest time and the length of the storage period under four methods. The Puerto Valle population produced seeds of good quality which conserved the viability for thirty-one weeks when they were stored with endocarp, in glass bottles.
\end{abstract}

Key words: Calophyllum brasiliense, seeds, storage, conservation, physiological quality.

\footnotetext{
1 Centro de Investigación y Desarrollo Tecnológico (CIDET). Facultad de Ciencias Exactas, Químicas y Naturales (FCEQyN). Félix de Azara 1552. 3300. Posadas. Misiones. csorol@fceqyn.unam.edu.ar

${ }^{2}$ Comité Ejecutivo de Desarrollo e Innovación Tecnológica. (CEDIT). Félix de Azara 1890 - 5to Piso. Posadas. Misiones. 3300. Argentina.sofi_cc22@hotmail.com

${ }^{3}$ CIDET. FCEQyN. Félix de Azara 1552. 3300. Posadas. Misiones. vercasol@gmail.com

${ }^{4}$ CIDET. FCEQyN. Félix de Azara 1552. 3300. Posadas. Misiones. naikelu@hotmail.com

${ }^{5}$ CIDET. FCEQyN. Félix de Azara 1552. 3300. Posadas. Misiones. faby_eckers@hotmail.com
} 


\section{INTRODUCCIÓN}

Calophyllum brasiliense Cambess (Calophyllaceae), "arary", se distribuye desde México hasta Argentina (Rodríguez, 2006). En este país, en las provincias de Misiones y Corrientes, forma poblaciones en los bordes de esteros y en las selvas higrófilas del río Paraná, de las cuales una de la provincia de Misiones y dos de Corrientes se han descripto en cuanto a su estructura y dinámica (González \& Sorol, 2011; Rodríguez et al., 2011; Cardozo et al., 2013), y están caracterizándose genéticamente (Bich et al., 2010; Percuoco et al., 2010; Talavera et al., 2012; Percuoco et al., 2013 a, b y c; Percuoco et al., 2014). Estas poblaciones forman parte de ecosistemas fragmentados, y las de la margen del río han reducido su tamaño por asociación a las cotas de inundación del embalse Yacyretá, lo cual las ha llevado a encontrarse en riesgo de extinción local (Rodríguez et al., 2009).

C. brasiliense Cambess es un árbol perennifolio, cuya altura oscila entre 25 a $40 \mathrm{~m}$, con copa redondeada y densa y tronco único. $\mathrm{Su}$ corteza grisácea y áspera presenta surcos longitudinales y profundos y sus hojas son simples, decusadas, pecioladas, coriáceas y de tamaño variable (Vásquez-Yanes et al., 1999; Salazar et al., 2000; Flores, 2002). Se trata de plantas dioicas y polígamas que presentan flores bisexuales y estaminadas, agrupadas en panículas axilares pedunculadas (Vásquez-Yanes et al., 1999). Las flores hermafroditas son epíginas, el ovario es unilocular y contiene un solo óvulo (Flores, 2002). Posee drupas globosas u ovoides. Cada fruto contiene una sola semilla (Vásquez-Yanes et al., 1999; Flores, 2002; Nery, 2006). El embrión, que ocupa toda la cavidad de la semilla, es recto, de color blanco o amarillo crema, con dos cotiledones gruesos, carnosos y almacenadores. La germinación es hipógea y la plántula criptocotiledonar (Flores, 2002; Jesús et al., 2014).

La floración y la fructificación son anuales y varían acorde a la distribución geográfica, dependiendo principalmente de la orografía y del clima (Cordero \& Boshier, 2003). En Argentina fructifican desde abril a septiembre (Sorol \& González, 2011).

Los frutos del "arary" forman parte de la dieta de insectos, pájaros y pequeños mamíferos (Salazar et al., 2000; Ribeiro et al., 2005) y en algunos países se emplean para alimentar al ganado (Cordero \& Boshier, 2003). Por otra parte, se han atribuido a este árbol numerosas propiedades medicinales (Huerta Reyes et al., 2004; Gasparotto et al., 2005; Kimura et al., 2005; Ibáñez, 2007; Souza et al., 2009; Castañeda et al., 2011; Gómez-Cansino et al., 2013; Verján et al., 2013; García-Zebadúa et al., 2014) a la vez que se lo aprecia por la madera empleada en construcción, ebanistería y para la obtención de fibras con las que se elabora pulpa para papel (Jøker \& Salazar, 2000; Flores, 2002; Nery, 2006).

Debido al valor de la especie y a la situación actual de las poblaciones argentinas, se iniciaron acciones de restauración y estudios de preservación de la calidad de sus semillas teniendo en cuenta las recomendaciones de Pezoa (2001) respecto a que las estrategias de conservación deben ser complementarias.

Las semillas de $C$. brasiliense fueron consideradas recalcitrantes tropicales y como tales no toleran la desecación a bajo contenido de agua $(<12 \%)$, ni el almacenamiento a baja temperatura (Bonner \& Vozzo, 1990; Ellis et al., 1990; Hong et al., 1996; Sacandé et al., 2004). Este tipo de semillas, propias de especies que habitan en ambientes muy húmedos, no experimentan deshidratación en la planta madre y se diseminan metabólicamente activas; sin detener su desarrollo germinan y se establecen como plántulas casi de inmediato (Kainer et al., 1999; Vásquez et al., 2004; Magnitskiy \& Plaza, 2007). No obstante ocurren algunos casos de latencia, las semillas recalcitrantes pierden rápidamente su capacidad de germinación al quedar expuestas a condiciones de baja humedad. Los mecanismos fisiológicos y bioquímicos de la intolerancia a la desecación no están completamente elucidados, lo cual dificulta la elección de métodos adecuados para conservarlas. Si bien existen técnicas para almacenar algunas semillas recalcitrantes por un corto período, cada especie requiere un método propio que debe ser estudiado (Farrant et al., 1993, Kermode \& FinchSavage, 2002; Fonseca \& Freire, 2003; Berjak \& Pammenter, 2004; Rao et al., 2007; Costa, 2009).

Los estudios sobre el almacenamiento de las semillas de $C$. brasiliense indican temperaturas de almacenamiento que van de $0^{\circ} \mathrm{C}$ a más de $15^{\circ} \mathrm{C}$ (Vásquez Yanes et al., 1999; Jøker \& Salazar, 2000; Silva, 2005) y contenido de humedad entre $25 \%$ y $35 \%$ aunque el contenido de humedad de 
las semillas recién cosechadas sea alto, pudiendo alcanzar un 60\% (Carvalho et al., 2006; Nery, 2006; Lima, 2011). Todos los trabajos citados evaluaron la capacidad germinativa teniendo en cuenta la protrusión de la radícula sin embargo no hacen referencia al establecimiento de plántulas.

La determinación del contenido de humedad de las semillas, al igual que su almacenamiento, requiere una metodología particular. Cualquiera sea el método empleado debe reducir la oxidación, la descomposición y la pérdida de otras sustancias volátiles a la vez de asegurar la mayor remoción de agua, por ello debe tener en cuenta las características de las semillas. Para semillas oleaginosas ISTA (1999) recomienda el método de estufa a baja temperatura y para las de gran tamaño indica que deben cortarse antes de ser secadas, a menos que su alto contenido de aceite lo dificulte. Al respecto las de C. brasiliense contienen 38\% a 39\% de lípidos (Nery et al., 2007) y son grandes, de 1,8 a $2,3 \mathrm{~cm}$ (Flores, 2002). Considerando estas cualidades y que no hay un modo estandarizado para esta especie es necesario establecer un método eficiente para la determinación del contenido de humedad de los embriones.

Los objetivos de este trabajo fueron evaluar la calidad de las semillas de $C$. brasiliense de las poblaciones argentinas, determinar un método adecuado para estimar el contenido de humedad y establecer un protocolo para el almacenamiento de las semillas que permita la conservación de la calidad fisiológica por tiempo prolongado.

\section{Materiales y Métodos}

\section{Evaluación de las poblaciones}

Entre los años 2009 y 2012 se estudiaron tres poblaciones de $C$. brasiliense que se describen a continuación.

a) Población de San Ignacio (SI), situada en la Reserva Privada "Luis Jorge Velásquez" dentro del Club de Pesca y Deportes Náuticos,

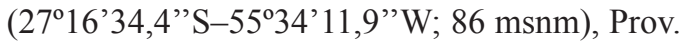
Misiones: Dpto. San Ignacio; forma parte de un fragmento de selva ribereña y está compuesta por aproximadamente 390 árboles/ha, dispuestos sobre 2 ha y altura media de los individuos de $10 \mathrm{~m}$ (Rodríguez et al., 2009).

b) Población de Rincón Ombú (RO), (2725'38”S-56¹6.27’'W; 88-82 msnm), Prov.
Corrientes: Dpto. Ituzaingó, a la vera del río Paraná, de aproximadamente 1 ha .

c) Población de la Estancia "Puerto Valle", (PV), ( $27^{\circ} 36^{\prime} 48^{\prime \prime}$ S $-56^{\circ} 30^{\prime} 48,6^{\circ} \mathrm{W}, 85 \mathrm{~m}$ s. n. m.), Prov. Corrientes: Dpto. Ituzaingó. En el noroeste de los esteros del Iberá, próxima a las plantaciones forestales de la empresa Pomera S.A.; fragmento de aproximadamente 2 ha (Fig. 1).

Los sitios de estudio se visitaron una vez al mes y los árboles que producían frutos se identificaron mediante la utilización de binoculares y se marcaron. Los frutos se cosecharon de los árboles con tijera con pértiga, y también manualmente del suelo conforme a lo sugerido por Flores (2002) luego se mezclaron. En cada una de las cosechas los frutos presentaban coloración verde clara y el tamaño se correspondía con el que normalmente presentan los frutos maduros de esta especie.

En el laboratorio se contaron los frutos y se eliminaron aquellos que se encontraban deteriorados. Luego se los despulpó manualmente hasta obtener las semillas contenidas en el endocarpo, se eliminaron aquellas cuyo endocarpo presentaba daño o signos de haber sido atacadas por insectos. Con la ayuda de una pinza se quebró el endocarpo, al cual quedaron adheridos los tegumentos seminales, se extrajeron los embriones y luego de descartar los oxidados y dañados se

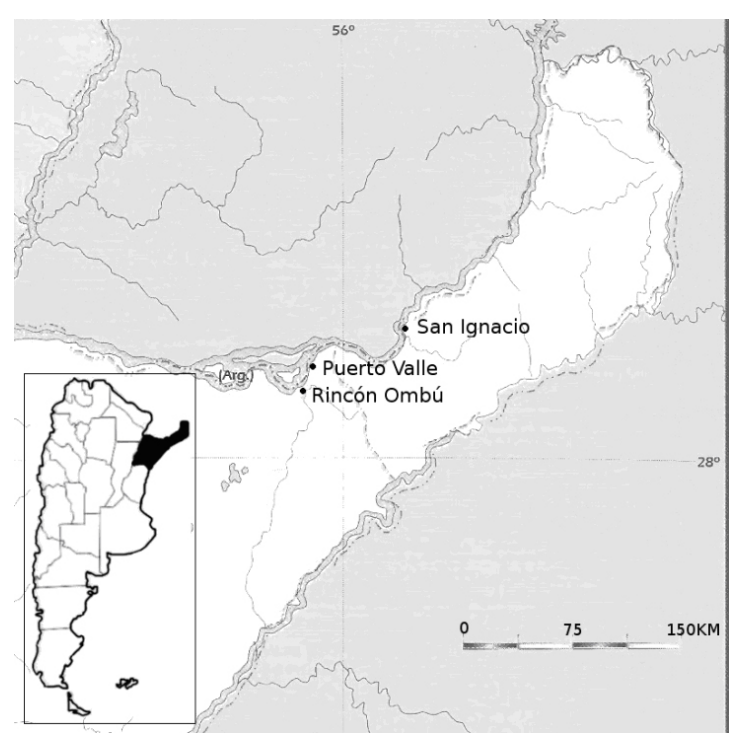

Fig. 1. Localización de las poblaciones de Calophyllum brasiliense. 
registró el número de embriones sanos. La ruptura del endocarpo permitió identificar las semillas vanas, que también se eliminaron.

Inmediatamente después de procesados los frutos, se evaluó la capacidad de germinación, para ello se dispusieron los embriones entre arena contenida en bandejas de plástico transparente que se expusieron a $8 \mathrm{~h}$ de luz y $25^{\circ} \mathrm{C}$. Se llevaron a cabo cuatro repeticiones de 25 embriones.

Semanalmente se contaron las plántulas normales, y el establecimiento de las mismas fue el criterio para considerar las semillas germinadas. Para calificar como normal a una plántula se tuvo en cuenta las Reglas Internacionales para los Ensayos de Semillas (ISTA, 1999) según las cuales una plántula normal debe ser capaz de continuar su desarrollo en planta normal cuando se la cultiva en suelo de buena calidad y bajo condiciones favorables. Se calculó el poder germinativo (PG) expresado como la proporción porcentual de semillas que germinaron en condiciones ideales.

\section{Análisis de la relación tamaño de la planta madre y cantidad de frutos producidos}

Se realizó en 2010 con material procedente de SI. En el campo se midió la circunferencia del tallo a la altura del pecho (CAP) y se cosecharon los frutos manteniéndolos separados por individuo los cuales luego se contaron en el laboratorio. Se evaluó la existencia de correlación entre la CAP y la cantidad de frutos producidos a través del análisis del coeficiente de Pearson $(P<0,05)$ empleando el paquete estadístico Statgraphics (2009).

Comparación de los métodos para determinar el contenido de humedad de los embriones de $C$. brasiliense

Se realizó en 2011 con material procedente de PV. Se trabajó con embriones extraídos de semillas procedentes de frutos cosechados dos días antes y se evaluaron dos factores, la temperatura y el modo de procesar los embriones, cada uno con dos niveles. Las temperaturas probadas fueron $105^{\circ} \mathrm{C}$ por $24 \mathrm{~h} \mathrm{y} 130^{\circ} \mathrm{C}$ por $1 \mathrm{~h}$; y se pusieron embriones enteros y embriones cortados; de la combinación de los niveles de los factores se obtuvieron cuatro tratamientos que se detallan a continuación: $105^{\circ} \mathrm{C}$ por $24 \mathrm{~h}$ con embriones enteros y con embriones cortados y $130^{\circ} \mathrm{C}$ por $1 \mathrm{~h}$ (método de estufa a alta temperatura) con embriones enteros y embriones cortados. El contenido de humedad $(\mathrm{CH})$ se estimó a partir de la diferencia de peso al comienzo y final del procedimiento según la fórmula: $\mathrm{CH}=($ peso inicial-peso final)/(peso inicial- peso del recipiente). Se hicieron tres repeticiones de cinco embriones. Luego se analizaron los resultados a través de un ANOVA $(P<0,05)$.

Evaluación del contenido de humedad de los embriones extraidos de semillas procedentes de frutos de diferentes cosechas

En PV, de abril a agosto de 2011, una vez al mes, se cosecharon frutos del suelo, del tamaño de los frutos maduros y de coloración verde clara lo cual indicaba que se habían desprendido recientemente de la planta madre.

Luego se determinó el $\mathrm{CH}$ mediante el método de estufa a alta temperatura con embriones enteros.

Evaluación del poder germinativo en relación al contenido de humedad

Se emplearon frutos cosechados en agosto de 2011 en PV. Para ello se realizó una evaluación inicial del CH y del PG inmediatamente después de la extracción de los embriones, luego se los puso entre capas de silicagel y cada 5 días se evaluaron las mismas variables.

El CH se determinó por el método de estufa a alta temperatura con embriones enteros y el PG a través de un ensayo de germinación, ambos realizados y evaluados del modo descripto.

Se calculó el coeficiente de correlación de Pearson para estimar la asociación entre el PG y el CH.

\section{Evaluación de las condiciones de almacenamiento}

Se emplearon frutos cosechados en 2012 en PV. Evaluación inicial: se determinó el $\mathrm{CH}$ inicial y el PG inicial de embriones extraídos de semillas recién colectadas. El $\mathrm{CH}$ se determinó por el método de estufa a alta temperatura con embriones enteros y para estimar la capacidad de germinación se sembraron embriones según las condiciones ya descriptas, se realizaron tres repeticiones de 25 embriones. Se evaluó el PG, la velocidad de germinación a través del índice de velocidad de germinación (IVG) que se calcula mediante la sumatoria de los cocientes que resultan de dividir el número de semillas germinadas por el tiempo al cual germinaron (Maguire, 1962) y el T50 
considerando el número de días al cual se registra el $50 \%$ de la germinación. Con el fin de identificar un método de almacenamiento que permita prolongar la viabilidad de las semillas de $C$. brasiliense se realizaron las siguientes evaluaciones.

a) Evaluación del efecto del recipiente en el almacenamiento de semillas. Se almacenaron semillas con endocarpo en bolsas de polietileno de baja densidad y $25,67 / \mathrm{m}^{2}$ de gramaje, y tamaño $15 \times 10 \mathrm{~cm} ; 100$ semillas por bolsa y semillas con endocarpo en frascos de vidrio con $300 \mathrm{~cm}^{3}$ de capacidad, 100 semillas por frasco.

b) Evaluación del efecto del recipiente en el almacenamiento de embriones. Se dispusieron los embriones en bolsas de polietileno de iguales características que las mencionadas. También se almacenaron embriones en bolsas de papel de $7,5 \times 13,5 \mathrm{~cm}$. En cada recipiente se guardaron los embriones extraídos de 100 semillas.

c) Evaluación del efecto del endocarpo. Se comparó el efecto de almacenar en bolsas de polietileno las semillas con endocarpo y los embriones.

Todos los recipientes fueron almacenados en heladera a $15 \pm 2^{\circ} \mathrm{C}$ hasta el momento de la siembra. Cada 15 días se tomaron muestras y se realizaron ensayos de germinación. En los casos en que se almacenaron semillas con endocarpo los embriones se extrajeron inmediatamente antes de la siembra. Al momento de hacerlo se advirtió que algunas semillas eran vanas y otras contenían embriones afectados por larvas o por oxidación. Por ello se estimó el porcentaje de pérdida y el porcentaje real de germinación calculado como el producto del PG por el porcentaje de embriones en buenas condiciones, sobre 100 .

Los resultados de cada tratamiento se analizaron mediante ANOVA considerando el tiempo de almacenamiento y cuando se encontraron diferencias significativas se compararon las medias con el Test de Duncan. Se evaluó la asociación entre las variables PG, IVG y T50 en función del tiempo a través del coeficiente de correlación de Pearson $(P<0,05)$.

\section{Evaluación de las plántulas anormales}

Fueron consideradas plántulas anormales aquellas que presentaron estructuras esenciales irreparablemente defectuosas que le impidieran desarrollarse en una planta normal (Bekendam \& Grob, 1979). Durante el experimento se registró la cantidad de plántulas anormales establecidas y se las caracterizó.

\section{Resultados}

\section{Evaluación de las poblaciones}

A continuación se describen las características de las poblaciones.

a) Población SI. Los individuos produjeron frutos desde abril a septiembre en forma desigual. En 2009, se identificó un total de 13 ejemplares que producían frutos. El PG fue de 67\%. En 2010 se encontraron ocho individuos produciendo frutos. En abril se cosecharon frutos del suelo pero al momento de extraer el embrión se halló que no estaban completamente desarrollados. La colecta fue sumamente escasa hasta septiembre, mes en el que de los ocho árboles se cosecharon 1259 frutos, de los cuales se obtuvieron 342 (27\%), embriones sanos, completos y sin evidencia de oxidación. El PG fue de $77 \%$.

b) Población RO. En 2009 el fragmento de Rincón Ombú estaba compuesto por 20 árboles en fructificación, que habían producido abundante cantidad de frutos y presentaba un rico banco de plántulas. Para igualar al número de individuos de la población SI se evaluaron 13 árboles de los que se recolectaron 2272 frutos. El PG fue de $72 \%$. A inicios de 2010 y pese a la altitud del terreno, $82 \mathrm{~m}$ s. n. m., esta población se encontraba seriamente afectada por la inundación y los ejemplares sobrevivientes produjeron muy pocos frutos.

c) Población PV. En este lugar muchas zonas del suelo son pantanosas y otras muy húmedas. En el transcurso de las diferentes visitas fueron identificándose árboles muy altos cuyas ramas productoras de frutos superaban la altura de la pértiga lo que determinó que la cosecha se realizara del suelo. Se observó un rico banco de plántulas. El PG fue de $79 \%$ en 2011 y $95 \%$ en 2012.

\section{Análisis de la relación tamaño del árbol y cantidad de frutos producidos}

En la tabla 1, se presenta la CAP de los individuos, el número total de frutos por árbol y la cantidad de embriones sanos extraídos. La correlación entre el tamaño de los árboles, expresado a través de la CAP, y la cantidad de frutos producidos no fue significativa $(r=0,42 ; P=0,05)$. 
Tabla 1. Características de los árboles de Calophyllum brasiliense de la población SI. Septiembre de 2010.

\begin{tabular}{|ccc|}
\hline $\begin{array}{c}\text { CAP } \\
(\mathbf{c m})\end{array}$ & Número de frutos & $\begin{array}{c}\text { Número de } \\
\text { embriones sanos }\end{array}$ \\
\hline 102 & 63 & 32 \\
160 & 283 & 71 \\
223 & 128 & 31 \\
73 & 146 & 31 \\
95 & 118 & 80 \\
184 & 332 & 36 \\
130 & 141 & 37 \\
133 & 48 & 24 \\
\hline
\end{tabular}

Solo de un $27 \%$ de los frutos se obtuvieron embriones sanos, completos y sin evidencia de oxidación, aunque al momento de la colecta todos tenían buena apariencia.

Comparación de los métodos para determinar el contenido de humedad de los embriones de C. brasiliense

Todos los tratamientos son útiles para determinar el $\mathrm{CH}$ en embriones de $C$. brasiliense. No se detectó diferencia en $\mathrm{CH}(F=0,06)$ (Fig. 2).

Evaluación del contenido de humedad de los embriones extraídos de semillas procedentes de frutos de diferentes cosechas

En las visitas realizadas en el transcurso del año 2011 se cosecharon frutos cuyo $\mathrm{CH}$ iba disminuyendo. No obstante los frutos cosechados en abril y en mayo aparentaban estar maduros, los

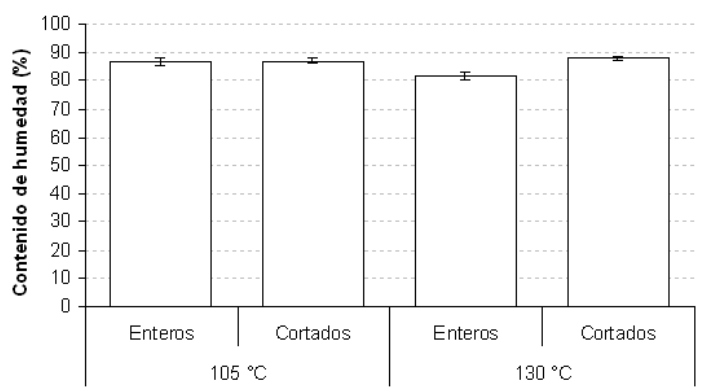

Fig. 2. Contenido de humedad (\%) de los embriones de Calophyllum brasiliense determinado con distintos métodos. embriones eran pequeños con aspecto vítreo, la coloración no era blanquecina opaca propia de los embriones maduros, y el $\mathrm{CH}$ era sumamente alto (Fig. 3).

Evaluación del poder germinativo en relación al contenido de humedad

El CH inicial fue de $41,79 \%$ y el $P G$ de $79 \%$. Conforme iba descendiendo el $\mathrm{CH}$ de los embriones el PG también lo hacía y fue nulo cuando el $\mathrm{CH}$ se aproximó a 19\% (Fig. 4) Las variables $\mathrm{PG}$ y $\mathrm{CH}$ están fuertemente asociadas $(r=0,97 ; P=0,05)$.

Evaluación de las condiciones de almacenamiento

Evaluación inicial: de las semillas tomadas para este ensayo, se llevó a germinación el $81 \%$ de los embriones extraídos, dado que el 19\% restante estaba compuesto por embriones atacados por

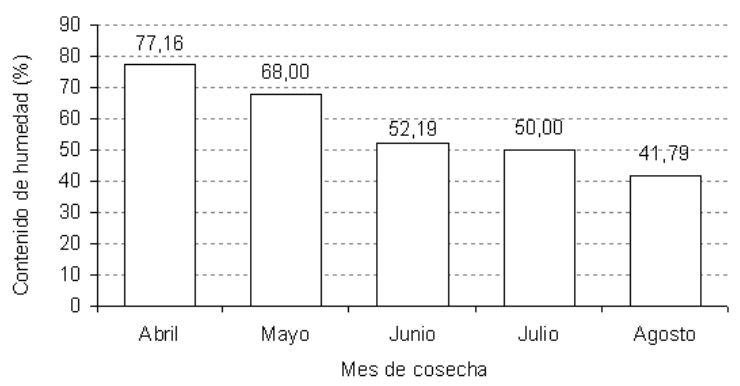

Fig. 3. Contenido de humedad (\%) de los embriones de Calophyllum brasiliense en las distintas cosechas.

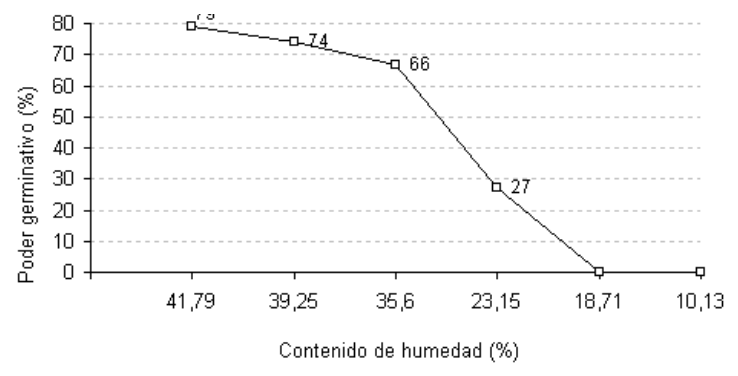

Fig. 4. Germinación de los embriones de Calophyllum brasiliense en relación al contenido de humedad. 
larvas, embriones infectados por hongos y por semillas vanas. El $\mathrm{CH}$ de los embriones fue de $27,67 \%$. Las plántulas se establecieron a partir de los 24 días de modo rápido, tal como señala el IVG de 0,5 y el T50 registrado a los 39 días. E1 PG fue de $95 \%$ y el porcentaje real de germinación de $77 \%$.

a) Evaluación del efecto del recipiente en el almacenamiento de semillas. El PG inicial fue de $95 \%$, el IVG inicial de 0,5 y el T50 se registró a los 39 días.

Los embriones extraídos de semillas almacenadas con endocarpo en bolsas de polietileno fueron evaluados hasta la $31^{\mathrm{a}}$ semana de almacenamiento. El porcentaje de embriones sanos en condiciones de ser llevados a ensayo de germinación fue disminuyendo hasta llegar a $50 \%$ en la semana $31^{\text {a }}$ debido a la oxidación, la infección o la presencia de semillas que habían sido almacenadas vanas.

El PG se mantuvo hasta la segunda semana, a partir de ese momento descendió en forma gradual hasta la $21^{\mathrm{a}}$ semana para luego decaer notoriamente $\left(F_{11,21}=15,56 ; P<0,05\right)$ (Fig. 5).

El IVG también se mantuvo hasta la segunda semana $\left(F_{11: 21}=9,12 ; P<0,05\right)$ (Fig. 6), después fue disminuyendo de modo constante tal como también lo evidenció el T50 que alcanzó su máximo valor, 77 días, en la última evaluación. El PG y el IVG se encontraron inversa y significativamente asociados al tiempo de almacenamiento como lo indican los coeficientes de correlación $(r=-0,88$

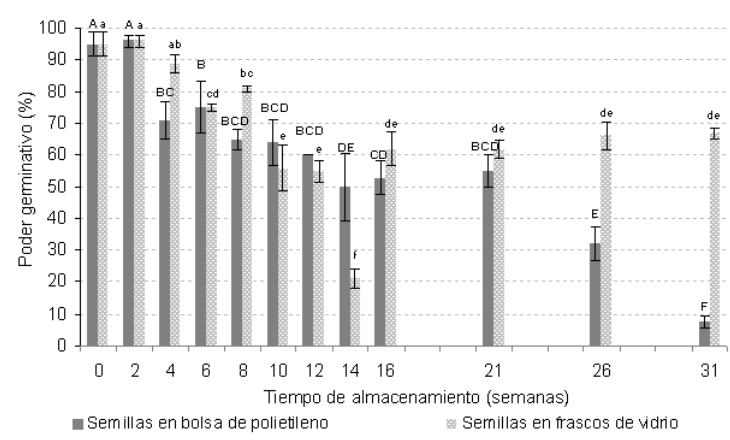

Fig. 5. Germinación de semillas de Calophyllum brasiliense almacenadas en bolsas de polietileno y en frascos de vidrio. Letras distintas indican diferencias significativas $(P<0,05)$ según Duncan. en ambos casos). Por otra parte el T50 se encontró, directa y moderadamente asociado al tiempo $(r=0,57)$.

Al igual que con el tratamiento anterior, las semillas almacenadas con endocarpo en frascos de vidrio pudieron ser evaluadas luego de treinta y un semanas de almacenamiento. El porcentaje de embriones en condiciones de ser evaluados fue disminuyendo al transcurrir el almacenamiento, y al llegar a la última evaluación había 27\% menos debido a las causas ya mencionadas. En este caso el PG se mantuvo hasta la cuarta semana, después fue disminuyendo con oscilaciones $\left(F_{11,21}=26,57\right.$; $P<0,05)$ (Fig. 5), hasta llegar a $67 \%$ en la última evaluación. Por su parte, el IVG se mantuvo hasta la octava semana de almacenamiento $\left(F_{11,21}=14,74\right.$; $P<0,05)$ (Fig. 6), pero luego la germinación fue tornándose más lenta y en la última evaluación el T50 se registró a los 73 días. El PG se asoció inversa y moderadamente al tiempo de almacenamiento como lo indica el coeficiente de correlación ( $r=$ $-0,62)$. El mismo tipo de asociación se encontró en el IVG $(r=-0,75)$. El T50 aumentó en función del tiempo de almacenamiento $(r=0,53)$.

b) Evaluación del efecto del recipiente en el almacenamiento de embriones. El PG inicial fue de $95 \%$, el IVG inicial de 0,5 y el T50 se registró a los 39 días.

El PG y el IVG de los embriones almacenados en bolsas de polietileno se mantuvieron hasta la décima semana de almacenamiento $\left(F_{8,14}=7,42 ; P<0,05\right)$ (Fig. 7), luego para la $16^{\mathrm{a}}$

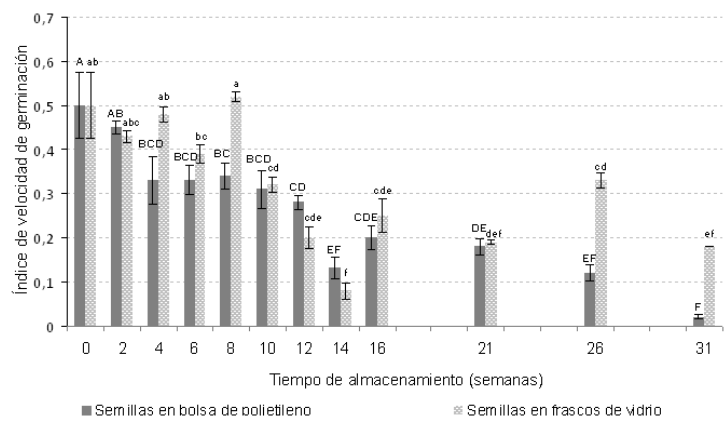

Fig. 6. Velocidad de germinación de semillas de Calophyllum brasiliense almacenadas en bolsas de polietileno y en frascos de vidrio.

Letras distintas indican diferencias significativas $(P<0,05)$ según Duncan. 


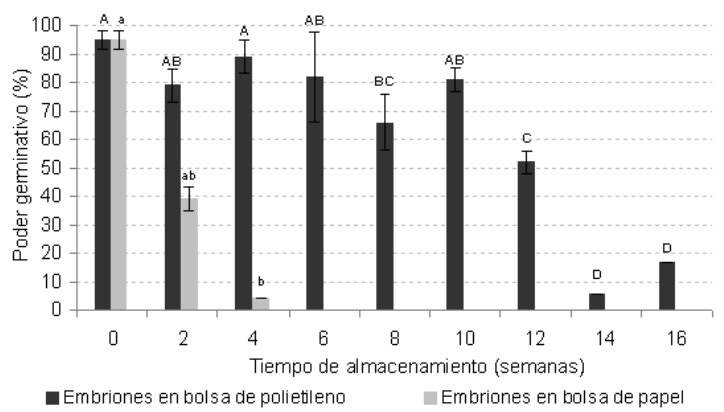

Fig. 7. Germinación de embriones de Calophyllum brasiliense almacenados en bolsa de polietileno y en bolsas de papel. Letras distintas indican diferencias significativas $(P<0,05)$ según Duncan.

semana el primero había disminuido un $64 \%$ $\left(F_{8,14}=32,26 ; P<0,05\right)$ (Fig. 8). En esta siembra, que fue la última, solo un $36 \%$ de los embriones almacenados pudo ser evaluado, debido a la infección que presentaban y que luego terminó por destruirlos. Ambas variables, PG e IVG estuvieron inversamente asociadas al tiempo $(r=-0,83$ y $r=0,64$ respectivamente). Por otra parte el T50 fue de 40 días y se asoció inversa y ligeramente al tiempo de almacenamiento $(r=-0,20)$.

Al almacenar embriones en bolsas de papel el PG se mantuvo por dos semanas, después descendió abruptamente y para la sexta semana los embriones ya no germinaron (Fig. 7). Esta variable estuvo asociada al tiempo de almacenamiento de modo moderadamente alto $(r=0,78)$. Al igual que

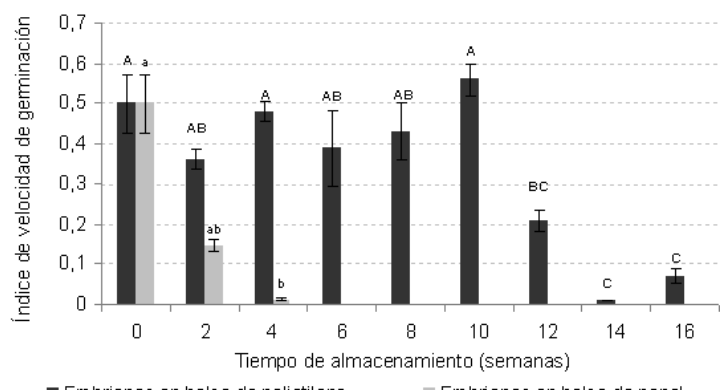

- Embriones en bolsa de polietileno

- Embriones en bolsa de papel

Fig. 8. Velocidad de germinación de embriones de Calophyllum brasiliense almacenados en bolsa de polietileno y en bolsas de papel. Letras distintas indican diferencias significativas $(P<0,05)$ según Duncan.

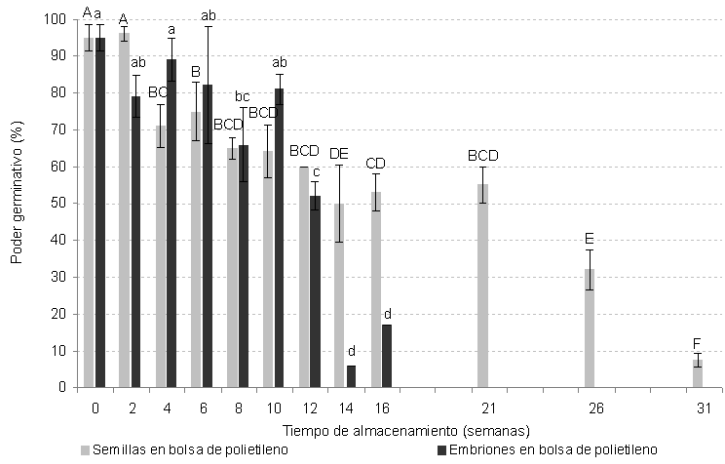

Fig. 9. Germinación de semillas y de embriones de Calophyllum brasiliense almacenados en bolsa de polietileno. Letras distintas indican diferencias significativas $(P<0,05)$ según Duncan.

el PG, el IVG decayó rápidamente (Fig. 8), tanto es así que después de cuatro semanas de estar almacenados, los embriones necesitaron 56 días para alcanzar el $50 \%$ de la germinación, es decir 16 días más con respecto al valor inicial. Al llegar a la octava semana el ensayo de germinación no pudo llevarse a cabo debido a que el $100 \%$ de los embriones estaba oxidado y extremadamente deshidratado.

c) Evaluación del efecto del endocarpo. A las 16 semanas de almacenamiento en bolsas de polietileno el porcentaje de germinación de las semillas con endocarpo fue de $53 \%$ y el IVG de 0,2 (Fig. 9 y 10), mientras que para embriones fue de $17 \%$ y 0,07 respectivamente. Esta fue la última evaluación de los embriones debido a la infección,

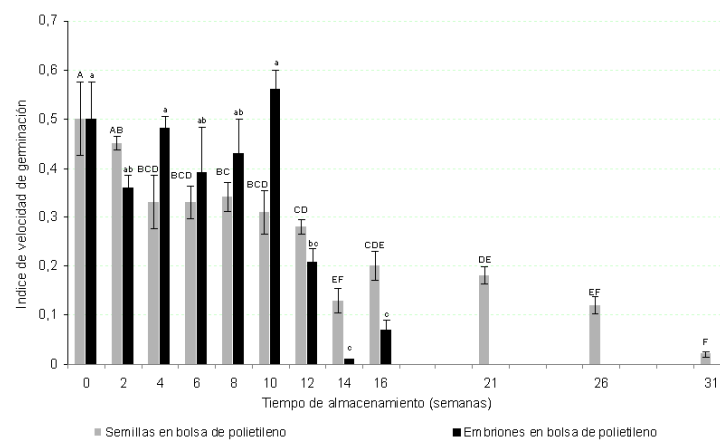

Fig.10. Velocidad de germinación de semillas y de embriones de Calophyllum brasiliense almacenados en bolsa de polietileno. Letras distintas indican diferencias significativas $(P<0,05)$ según Duncan. 


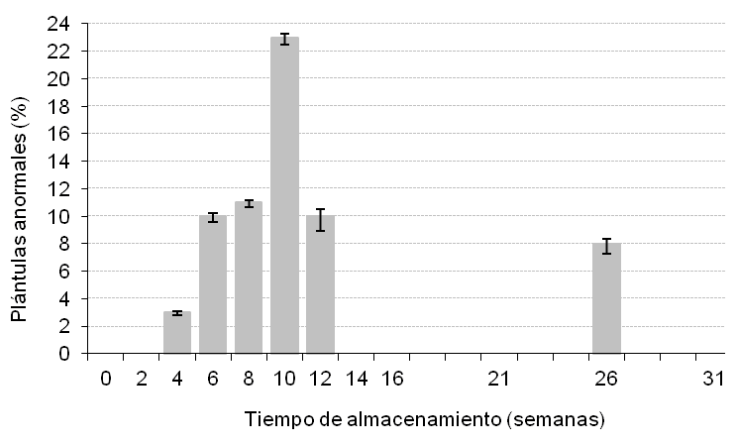

Fig. 11. Plántulas anormales de Calophyllum brasiliense en función del tiempo de almacenamiento.

sin embargo las semillas con endocarpo pudieron ser evaluadas hasta la $31^{\text {a }}$ semana aunque el PG fue menor al $10 \%$.

\section{Evaluación de las plántulas anormales}

Exceptuando el tratamiento en el que los embriones se almacenaron en bolsas de papel,

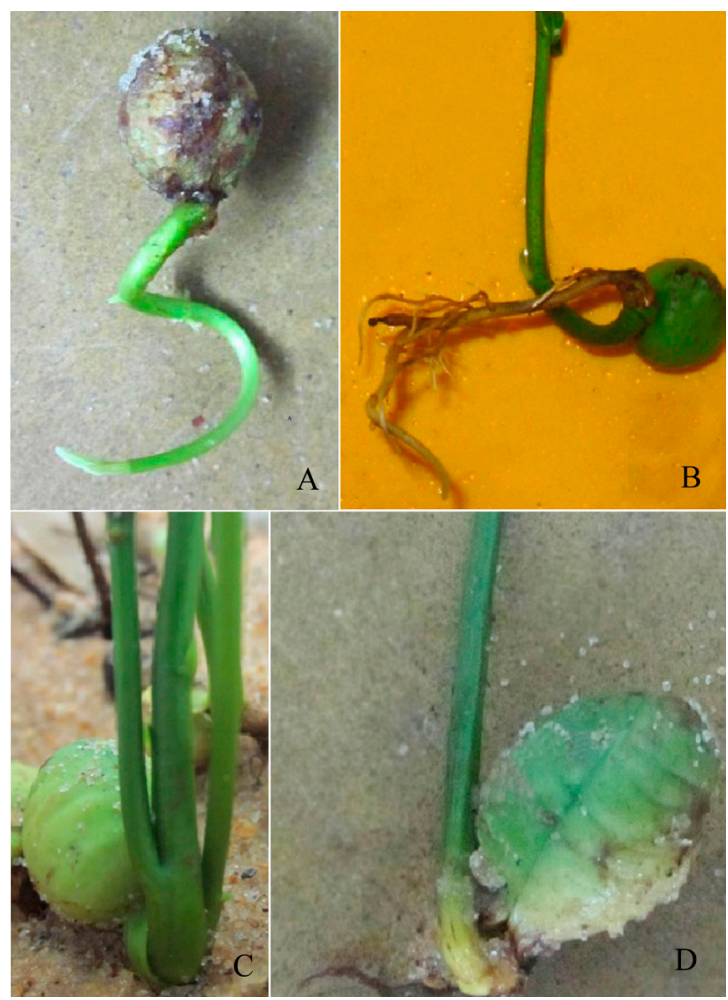

Fig. 12. Plántulas anormales establecidas en los ensayos de germinación. A y B hipocótilo en lazo. C. Presencia de varios vástagos. D. Ausencia de raíz. en los demás empezaron a establecerse plántulas morfológicamente anormales luego de cuatro semanas de almacenamiento y su número aumento hasta alcanzar un $23 \%$ después de un almacenamiento de diez semanas (Fig. 11). Las anormalidades consistieron en presencia de varios epicótilos (hasta tres inclusive), epicótilo formando lazo y raíces atrofiadas o ausentes (Fig. 12). También se observó una plántula albina.

\section{Discusión}

No obstante el cronograma de llenado del embalse preveía alcanzar la cota 82 a fines de 2010 la población de RO quedó prácticamente bajo agua en 2009; la de SI disminuyó de tamaño progresivamente y algunos individuos que producían frutos dejaron de hacerlo o quedaron en zonas netamente inundadas. Por su parte la población de $\mathrm{PV}$, que se localiza próxima al inicio de los esteros del Iberá, continuó produciendo frutos.

Flores (2002) señaló para $C$. brasiliense que el DAP de los árboles de los cuales se colecten frutos no debe superar los $70 \mathrm{~cm}$ (esto es CAP entre 126 y $220 \mathrm{~cm}$ ) porque al ser más grandes suelen presentar deterioro fisiológico, por ello se considera adecuado el tamaño de los individuos de SI. Además se constató que el tamaño, y en consecuencia la edad de los árboles, no influyó en la cantidad de frutos producidos, dado que esta variable no estuvo asociada a la CAP. Es posible que debido al disturbio que afectó a la población muchos factores que normalmente influyen en la calidad de los frutos estén ocupando un segundo plano o sean irrelevantes.

En muchas especies durante el proceso de maduración de los frutos ocurren modificaciones que incluyen cambios notorios de la coloración que permiten distinguir fácilmente a los frutos maduros (Dias, 2001). Este no es el caso de $C$. brasiliense cuyos frutos se mantienen verdes a la madurez, aunque de color menos brillante o verde-amarillentos (Bonner, 2008). En las poblaciones analizadas se cosecharon frutos color verde claro dado que esa coloración fue el criterio para considerarlos maduros. En el laboratorio la identificación de los frutos maduros fue más precisa y estuvo relacionada con la facilidad para 
quitar el mesocarpo (despulparlos), aunque Jøker \& Salazar (2000) indicaron que es el pericarpio en su totalidad el que puede retirarse fácilmente.

Otros indicios de madurez de los frutos suelen ser la liberación de la planta madre y el tenor de agua que contienen las semillas, aunque la madurez de los frutos no da la certeza de semillas en ese estado, de modo que estas pueden estar en proceso de maduración y mantener un elevado contenido de agua (Jara, 1996; Angón et al., 2006). En los estudios llevados a cabo por Lines et al. (2006) en C. brasiliense, cuando los frutos maduros comenzaron a caer de los árboles las semillas contenían alrededor del $60 \%$ de humedad, sin embargo en este estudio una humedad de $50 \%$ era muy alta y no se correspondía con un estado de madurez. Es posible que los frutos colectados del suelo en abril y mayo de 2011 hubieran caído por acción de fuertes vientos o tormentas y que en la secuencia de recolecciones se tomaron frutos en distintos estadios.

Para determinar el CH de las semillas las Reglas ISTA (1999) solo aprueban los métodos directos de estufa, por ello fueron los que se emplearon en este trabajo. Entre las variantes probadas la más eficiente fue la de utilizar embriones enteros a 130 ${ }^{\circ} \mathrm{C}$ durante $1 \mathrm{~h}$ dado que evita el trabajo cuidadoso que implica cortar los embriones sin que pierdan agua.

Teniendo en cuenta la relevancia del $\mathrm{CH}$ en la viabilidad de las semillas recalcitrantes se evaluó esta relación y se estableció que en $C$. brasiliense el $\mathrm{CH}$ próximo a $19 \%$ resultó letal, tal como afirma Jara (1996) para todas las semillas de este tipo. En los estudios realizados por Nery (2006) un $\mathrm{CH}$ próximo a $21 \%$ resultó tan solo crítico y a partir de allí la germinación comenzó a descender, en cambio Lines et al. (2006) establecieron que las semillas germinaban $(\mathrm{PG}=9 \%)$ incluso conteniendo $5 \%$ de agua, lo cual teniendo en cuenta los conceptos de Roberts (1973); Ellis et al. (1990); Hong et al. (1996) y Sacandé et al. (2004) las definiría como ortodoxas.

Nery et al. (2007) señalan que las semillas maduras de $C$. brasiliense tienen aproximadamente $45,6 \%$ de humedad y un porcentaje de germinación de $93 \%$, mientras que Vasquez et al. (2004) informaron para semillas de Costa Rica de la cosecha 1999 un $\mathrm{CH}$ de 43,5\% y porcentaje de germinación de $60 \%$; y para las de la cosecha
2000, 36\% de humedad y 90\% de germinación. Ellos destacaron que además de existir diferencias en la humedad de las semillas procedentes de frutos con el mismo grado de madurez, las semillas con bajo $\mathrm{CH}$ pueden presentar alto porcentaje de germinación siempre y cuando no hayan sido secadas. Así podría atribuirse la diferencia en PG de $27 \%$ en 2011 y $95 \%$ en 2012 para semillas con $\mathrm{CH}$ de $23,15 \%$ y $27,67 \%$ respectivamente, al hecho de que las primeras fueron secadas artificialmente.

El modo de evaluar los resultados en un ensayo de germinación debe estar relacionado con el objetivo general de la investigación, es así que se puede emplear un criterio botánico en el que se considera que una semilla ha germinado cuando se produjo la protrusión de la radícula o bien se opta por una regla tecnológica y se cuentan las plántulas normales. Evidentemente el modo elegido puede dar diferencia en los resultados, porque varios pueden ser los eventos que sucedan desde la aparición de la radícula hasta el establecimiento de un individuo. En este trabajo se evaluaron plántulas normales dado que complementa tareas de restauración en las que es necesario contar con nuevos individuos a ser reclutados a parcelas en el marco de una estrategia de conservación más amplia. Lines et al. (2006) consideró el mismo criterio al estudiar el almacenamiento de semillas. Ellos determinaron un $\mathrm{CH}$ de $21,30 \%$ y un $\mathrm{PG}$ próximo a $80 \%$ en semillas procedentes de frutos recién cosechados, luego las almacenaron en bolsas de plástico con perforaciones, dispuestas en cajas de plástico, a $15^{\circ} \mathrm{C}$ durante 6 meses y al cabo de ese tiempo lograron un PG de $8 \%$.

Por otra parte, la bibliografía hace referencia a porcentajes de germinación como variable para expresar la calidad de las semillas, pero habiendo detectado en este trabajo los problemas que presentan (semillas vanas y deterioradas) se considera que el porcentaje de germinación no estaría expresando la verdadera calidad del lote y por ello al momento de estimar la cantidad de material a cosechar y almacenar sería necesario tener en cuenta el porcentaje de pérdida de semillas y el porcentaje real de germinación En este estudio se determinó un porcentaje de pérdida inicial de 19\% que resultó elevado respecto al 11\% informado por Sánchez (1995).

Con relación al ensayo de almacenamiento, las 


\section{B. Sorol et al. - Conservación de semillas de Calophyllum brasiliense}

evaluaciones iniciales indicaron un $\mathrm{CH}$ de $27,67 \%$ y $\mathrm{PG}$ de $95 \%$, no obstante el porcentaje real de germinación fue de $77 \%$. Este resultado está próximo al obtenido por Vasquez et al. (2004), quienes informaron porcentajes de germinación de $68 \%$ y $90 \%$ en semillas con $40 \%$ y $34 \%$ de $\mathrm{CH}$ respectivamente. Cabe aclarar que este autor sembró embriones con endocarpo y al final del ensayo identificó vanas y deterioradas. Por su parte, Nery (2006) a partir de dos lotes con $\mathrm{CH}$ de $33,71 \%$ y $31,23 \%$, registró porcentajes de germinación de $83,9 \%$ y $89,4 \%$ respectivamente. $\mathrm{Si}$ bien estos valores son elevados, debe tenerse en cuenta que corresponden a plántulas con hipocótilo de $5 \mathrm{~mm}$ de longitud.

La temperatura es decisiva para lograr el éxito en el almacenamiento de las semillas recalcitrantes, este factor influye en concordancia con otros. Al respecto Salazar et al. (2000) indicaron que en el trópico, donde la temperatura oscila entre 20 y $30^{\circ} \mathrm{C}$ y la humedad relativa del aire es alta, las semillas de $C$. brasiliense pueden ser almacenadas a granel por dos a tres meses, sin embargo más allá de este período se deshidratan y el porcentaje de germinación baja drásticamente. Por su parte, Lines et al. (2006) establecieron que las semillas almacenadas a temperatura ambiente durante seis meses presentaron $12,3 \%$ de germinación. Vasquez et al. (2004) informaron que el almacenamiento a $15^{\circ} \mathrm{C}$ con $\mathrm{CH}$ de $31,8 \%$ fue el mejor tratamiento, aun así no lo pudieron extender más allá de seis meses. Por su parte Nery (2006) almacenó embriones durante nueve meses a $8^{\circ} \mathrm{C}$.

Teniendo en cuenta que el tipo de recipiente también influye en el almacenamiento, se consideró la propuesta de Nery (2006) y se almacenaron en bolsas de polietileno embriones con endocarpo. En esas condiciones la germinación ocurrió incluso después de treinta y un semanas de almacenamiento, aunque en bajo porcentaje, $7,5 \%$, pero como el material estaba deteriorado el porcentaje real de germinación fue de 3,7\%.

En las semillas recalcitrantes las cubiertas seminales ayudan a conservar la humedad (Magnitskiy \& Plaza, 2007) y C. brasiliense no es la excepción, dado que el almacenamiento de semillas con endocarpo se extendió 15 semanas más que el de embriones.

Respecto a la velocidad de germinación,
Vasquez et al. (2004) sólo indicaron que la germinación de $C$. brasiliense es lenta, pero Nery (2006) la determinó calculando el IVG y estableció que luego de tres meses de almacenar semillas en bolsa de polietileno el IVG era más alto que en otros tratamientos. En este estudio el resultado de la evaluación de esta variable asume importancia, dado que da cuenta del vigor de los embriones y permite afirmar que el almacenamiento de semillas en frascos de vidrio las mantuvo vigorosas hasta la octava semana de almacenamiento.

Sánchez (1995) informó que las semillas que han perdido humedad presentan germinación más lenta y producen plantas débiles o anormales. Ambos fenómenos se observaron en este trabajo. Se encontró mayor cantidad de plántulas anormales al almacenar semillas en frascos de vidrio, lo que estaría indicando que ocurrió deterioro gradual y que las semillas se encontraban en una etapa previa a la pérdida de viabilidad. En los otros tratamientos el deterioro ocurrió más rápido.

\section{Conclusiones}

En virtud de los resultados se destacan los siguientes aspectos:

- La población de la Estancia "Puerto Valle" produce semillas de buena calidad que al ser almacenadas conservan la viabilidad durante treinta y un semanas.

- Todos los tratamientos para determinar el $\mathrm{CH}$ en embriones de Calophyllum brasiliense son útiles.

- Para determinar el contenido de humedad, el método de estufa a $130{ }^{\circ} \mathrm{C}$ durante una hora con embriones enteros es el más eficiente en términos de trabajo y de tiempo.

- El contenido de humedad de los embriones de $18,71 \%$ resulta letal.

- La viabilidad de las semillas recalcitrantes de C. brasiliense se conserva hasta la $12^{\mathrm{a}}$ semana cuando son almacenadas a $15{ }^{\circ} \mathrm{C}$ en frascos de vidrio.

- El almacenamiento de las semillas en frascos de vidrio fue más efectivo que en bolsas de polietileno y permite conservar la calidad fisiológica por más tiempo.

- El almacenamiento de semillas con endocarpo fue más eficiente que el almacenamiento de embriones. 


\section{Agradecimientos}

A los Ingenieros Forestales Sebastián Robbiani, Guillermo Antonio Quinteros y Omar Sosa de la Empresa Pomera; al Sr. Roberto Musial de la Reserva Natural Privada Luis Jorge Velásquez y al Sr. Luis Krause del Club Refugio Ombú por permitirnos trabajar en los respectivos predios. Al Ing. Guillermo Antonio Quinteros y al Sr. Luis Krause por guiarnos en las primeras excursiones. Al Dr. Miguel Schmalko por las sugerencias para el análisis estadístico.

\section{Biblografía}

ANGÓN-GALVÁN, P., N. F. SANTOS SÁNCHEZ \& C. G. HERNÁNDEZ. 2006. Índices para la determinación de las condiciones óptimas de maduración de un fruto. Temas de ciencia y tecnología 10: 3-8.

BEKENDAM, J. \& R. GROB. 1979. Manual para evaluación de plántulas en análisis de germinación. Instituto Nacional de Semillas y Plantas de Vivero, Estación de Ensayo de Semillas. Zurich.

BERJAK, P. \& N. PAMMENTER. 2004. Recalcitrant seeds. In: BEECH-ARNOLD, R. \& R. SÁNCHEZ (eds.), Handbook of seed physiology, pp. 305-345. Food Products Press, New York.

BICH, G. A., C. B. PERCUOCO, M. E. RODRÍGUEZ, A. E. CARDOZO, J. V. CRISCI, C. F. ARGÜELLES. 2010 Genetic characterization of arary (Calophyllum brasiliense Cambess.): an endangered forest tree population from San Ignacio, Misiones - Argentina. XIV Congreso de ALAG - XXXIX Congreso de la SAG. Viña del Mar. pp. 274- 275.

BONNER, F.T. \& J. A. VOZZO. 1990. Storing recalcitrant tropical forest tree seeds. In: Triviño, D.T.; Jara N., L. F. (eds.). Memorias del Seminario- Taller sobre investigaciones en semillas forestales tropicales. CONIF. Serie Doc. 18: 139-142. Bogotá.

BONNER, F.T. 2008. Woody Plant Seed Manual. Bonner, F.T. and R.P. Karrfalt (eds.), Seed biology. USDA Forest Service Agriculture Handbook 727: 3-38, Washington, DC.

CARDOZO, A. E.; C. B. SOROL, C. B. PERCUOCO, N. L. GONZÁLEZ \& M. E. RODRÍGUEZ. 2013. Estructura y Dinámica de Calophyllum brasiliense Cambess. (Calophyllaceae) arary. Jornadas Científico-Tecnológicas; $1^{\mathrm{a}}$ ed. Posadas: EdUNaM. p. 211

CARVAlHO, I., E. SILVA \& A. DAVIDE. 2006. Classificação de sementes florestais quanto ao comportamento no armazenamento. Rev. Brasil. Sementes 28:7-14.
CASTAÑEDA, B., R. CASTRO, P. PUEBLA \& L. IBÁÑEZ. 2011. Efecto del extracto atomizado de las hojas de Calophyllum brasiliense "lagarto caspi" sobre la glicemia. Rev. Horizonte Médico 11: 7-14.

CORDERO, J. \& D. BOSHIER. 2003. Árboles de Centroamérica: un manual para extensionistas. Centro Agronómico Tropical de Investigación y Enseñanza.

COSTA, C. J. 2009. Armazenamento e conservação de sementes de espécies do cerrado. Nascimento, F. E. do \& Flores de Oliveira Arbués J. (eds.), $1^{\mathrm{a}}$ ed. Planaltina.

DIAS, C. F. 2001. Maduración de la semilla. Seeds news. (6), [online]. Disponible en: http://www.seednews. inf.br/espanhol/seed56/artigocapa56a_esp.shtml [Acceso: 15 Septiembre 2012].

ELLIS, R., T. HONG \& E. ROBERTS. 1990. An intermediate category of seed storage behaviour? I. Coffee. J. Exp. Bot. 41: 1167-1174.

FARRANT, J. M., N. W. PAMMENTER \& P. BERJAK. 1993. Seed development in relation to desiccation tolerance: a comparison between desiccation sensitive (recalcitrant) seeds of Avicennia marina and desiccation tolerant types. Seed Sci. Res. 3: 1-13.

FLORES, E. 2002. Calophyllum brasiliense. Trees and Seeds from Neotropics 3: 347-350.

FONSECA, S. L. \& H. FREIRE. 2003. Sementes recalcitrantes: Problemas na pos-colheita. Bragantia 62: 297-303.

GARCÍA-ZEBADÚA J. C., R. REYES-CHILPA, M. HUERTA-REYES, J. I. CASTILLO-ARELLANO, S. SANTILLÁN-HERNÁNDEZ, B. VÁZQUEZASTUDILLO, \& J. A. MENDOZA-ESPINOZA. 2014. El árbol tropical Calophyllum brasiliense: una revisión botánica, química y farmacológica. Rev. Fac. Quim. Farmacia 2: 126-145.

GASPAROTTO, A., M. BENZAN, I. PILOTO \& D. GARCIA-CROTEZ. 2005. Estudo fitoquímico da atividade moluscida de Calophyllum brasiliense Camb. (Clusiaceae). Quim. Nova 28: 575-578.

GÓMEZ-CANSINO, R., C. I. ESPITIA-PINZÓN, S. L. GUZMÁN-GUTIÉRREZ, M. G. CAMPOSLARA \& R. REYES-CHILPA. 2013. Actividad antimicobacteriana y anti-VIH-1 de especies de Clusiaceae y Julianaceae de México. En memoria digital del XXII Congreso de la sociedad italo latinoamericana de etnomedicina "Hernán Arguedas Soto”. Puntarenas. Costa Rica. pp. 235-236.

GONZÁlEZ, N. L. \& C. B. SOROL. 2011. Capacidad reproductiva de Calophyllum brasiliense Camb. Clusiaceae en Puerto Nuevo. I Congreso Nacional de Ciencias Agrarias. San Lorenzo. p. 52.

HONG, D., S. LININGTON \& R. ELLIS. 1996. Seed Storage Behaviour: A Compendium. Handbooks for Genebanks: № 4. IPGRI, Rome. 


\section{B. Sorol et al. - Conservación de semillas de Calophyllum brasiliense}

HUERTA-REYES, M., M. BASUALDO, F. ABE, M. JIMENEZ-ESTRADA, C. SOLER \& R. REYESCHILPA. 2004. HIV-1 Inhibitory Compounds from Calophyllum brasiliense. Leaves. Biol. Pharm. Bull. 27: 1471-1475.

IBÁÑEZ, V. L. 2007. Efecto antitumoral, anti VIH y elucidación estructural de las hojas y corteza de Calophyllum brasiliense de Satipo y Pucallpa. Rev. Acad. Perú Salud 14: 90-96.

ISTA, 1999. International rules for seed testing, 1999. Seed Sci. Technol. 27: 27-32 y 155-159.

JARA, L. 1996. Biología de semillas forestales. Centro Agronómico Tropical de Investigación y Enseñanza. Profesor: Danida Forest Seed Centre. Turrialba.

JESUS V. A. M. de, A.L. BRACCINI, L. A de SOUZA, I. S. MOSCHETA, G. E. GONÇALVES MARIUCCI \& F. L. SANTOS. 2014. Morphology and anatomy of the seedling and the tirodendro of Calophyllum brasiliense Cambess. (Clusiaceae). Acta Scientiarum Biol. Sci. 36: 443-449.

JØKER, D. \& R. SALAZAR. 2000. Calophyllum brasiliense Series: Seed Leaflets. CATIE 46: 2.

KAINER, K., M. DURYEA, M. MALAVASI, E. RODRIGUES DA SILVA \& J. HARRISON. 1999. Moist storage of Brazil nut seeds for improved germination and nursery management. Forest Ecol. Manag. 116: 207-217.

KERMODE, A. R. \& B. E. FINCH-SAVAGE. 2002. Desiccation sensitivity in orthodox and recalcitrant seeds in relation to development. In: BLACK, M. y H.W. PRITCHARD (eds.), Desiccation and survival in plants. Drying without dying, pp. 149184. CABI, Wallingford.

KIMURA, S., C. ITO, N. JYOKO, H. SEGAWA, J. KURODA, M. OKADA \& otros. 2005. Inhibition of Leukemic Cell Growth by a Novel Anti-Cancer Drug (GUT - 70) from Calophyllum brasiliense that acts by induction of apoptosis. Int. J. Cancer. 113:158-165.

LIMA, J. A. de. 2011. Padrão de germinação de sementes e establecimento de mudas de Guanandí (Calophyllum brasiliense Cambess.). (Tesis Doctoral) Univ. Federal Uberlândia. Minas Gerais.

LINES, K., J. HERRERA \& W. VÁSQUEZ. 2006. Estudio de la germinación y la conservación de semillas de Cedro María (Calophyllum brasiliense). Technol Marcha. 19: 61-72.

MAGNITSKIY, S. \& G. PLAZA. 2007. Fisiología de semillas recalcitrantes de árboles. Agronomía Colombiana 25: 96-103.

MAGUIRE, J. 1962. Speed of germination-aid in selection and evaluation for seedling emergence and vigor. Crop Sci. 2: 176-177.

NERY, F.C. 2006. Aspectos da germinação, armazenamento de sementes, crescimento inicial e anatomia de plantas jovens de Calophyllum brasiliense Cambess. (Tesis de Maestria). Univ. Federal de Lavras. Minas Gerais.

NERY, F., A. ALVES, C. JUSTO, E. CASTRO \& V. STEIN. 2007. Caracterizaçao Morfológica e Química de Sementes de Calophyllum brasiliense Camb. Rev. Bras. Bioci. 5:144-146.

PERCUOCO, C. B., L. N. TALAVERA STEFANI, M. E. RODRÍGUEZ, J. F. CRIVELLO, N. L. GONZALEZ, A. E. CARDOZO \& otros. 2013a. Filogeografía de poblaciones de Calophyllum brasiliense Camb. (Calophyllaceae) en el límite sur de su distribución. Bol. Soc. Argent. Bot. 48 (Supl.): 89.

PERCUOCO, C. B.; L. N. TALAVERA STÉFANI, J. V. CRISCI, C. F. ARGÜELLES. 2013b. Regiones no codificantes del genoma plastidial de Calophyllum brasiliense Camb. (Calophyllaceae) en poblaciones del noreste argentino. Jornadas CientíficoTecnológicas $1^{\mathrm{a}}$ ed. Posadas: EdUNaM p. 211.

PERCUOCO, C. B., L. N. TALAVERA STEFANI, M. E. RODRÍGUEZ, A. E. CARDOZO, N. L. GONZÁlEZ, C. B. SOROL \& otros. 2013 c. Relaciones filogeográficas entre poblaciones argentinas de Calophyllum brasiliense Camb. basadas en ADNcp. BAG. XLII Congreso Argentino de Genética - III Reunión Regional NOA. Salta. p. 193.

PERCUOCO, C. B., G. A. BICH, M. E. RODRÍGUEZ, A. E. CARDOZO, C. F. ARGÜELLES. 2010. Genetic characterization of a Calophyllum brasiliense Camb. (Clusiaceae) population from San Ignacio, MisionesArgentina. VI Southern Connection Congress. Bariloche.

PERCUOCO, C. B., G. A. BICH, TALAVERA STÉFANI, L. N.; N. L. GONZÁLEZ,M. E. RODRIGUEZ, A. E. CARDOZO, C. B. SOROL, J. F. CRIVELLO, J. V. CRISCI, C. F. ARGÜELLES. 2014. Evaluación genética rápida de poblaciones argentinas de Calophyllum brasiliense Camb. (Calophyllaceae) en riesgo de extinción local: recomendaciones para su conservación. I Congreso Latinoamericano de Genética para la Conservación. Macuto. p. 33.

PEZOA, A. 2001. Estrategias de Conservación de la diversidad biológica. En: SQUEO, F. A., G. ARANCIO \& J.R. GUTIÉRREZ (eds.), Libro Rojo de la Flora Nativa Sitios Prioritarios para su conservación, pp. 273-280. Univ. La Serena. La Serena.

RAO, N., J. HANSON, M. DULLOO, K. GHOSH, D. NOVELL \& M. LARINDE. 2007. Manual para el manejo de semillas en bancos de germoplasma. Biovers. Int. 8:147.

RIBEIRO MELLO, M. A., N. OLIVEIRA LEINER, P. R. GUIMARÃES JR. \& P. JORDANO. 2005. Sizebased fruit selection of Calophyllum brasiliense 
(Clusiaceae) by bats of the genus Artibeus (Phyllostomidae) in a Restinga area, southeastern Brazil. Acta Chiropterologica 7: 179-182.

ROBERTS, EH. 1973. Predicting the storage life of seeds. Seed Sci. Tech.1: 499-514.

RODRÍGUEZ, M. E., J. F. CRIVELLO, A. E. CARDOZO, N. L. GONZÁlEZ, C. F. ARGÜELlES, C. B. PERCUOCO \& otros. 2011. Selvas ribereñas con Calophyllum brasiliense, su status fitogeográfico en la Argentina. VIII Jornadas de Investigación Científico Tecnológicos de la Facultad de Ciencias Exactas, Químicas y Naturales de la Universidad Nacional de Misiones. Posadas. p. 44.

RODRÍGUEZ, M., A. CARDOZO, E. KRAUCZUK, J. FONTANA \& D. IRIART. 2009. Calophyllum brasiliense (Clusiaceae): nuevo registro para la flora de la Argentina. Bol. Soc. Argent. Bot. 44: 361-366.

RODRÍGUEZ, M., E. KRAWCZUK, A. CARDOZO, J. FONTANA \& D. IRIART. 2006. Calophyllum brasiliense Cambess. (Clusiaceae), una nueva especie para la flora argentina. 57 ${ }^{\circ}$ Congreso Nacional de Botánica., v. CD-rom. Gramado.

SACANDÉ, M., D. JØKER, M. DULLOO \& K. THOMSEN. 2004. Comparative storage biology of tropical tree seeds. IPGRI, Rome.

SALAZAR, R., C. SOIHET \& J. MÉNDEZ. 2000. Calophyllum brasiliense Cambes. En: Manejo de semillas de 100 especies forestales de América Latina. CATIE 58: 115-116.

SÁNCHEZ, J. 1995. Aspectos de la fisiología de la germinación y almacenamiento de semillas de importancia forestal. En: SALAZAR R. (ed.), Salazar, Memorias. Avances en la producción de Semillas Forestales en América Latina. pp. 165-168. CATIE.

SILVA, E. K. da 2005. Calophyllum brasiliense Cambess. Jacareúba. Info. Téc. Rede de Sementes da Amazônia 11.

SOROL, C. B. \& N. L. GONZÁLES. 2011. Obtención de plántulas de Calophyllum brasiliense, por rescate y a partir de semillas, destinadas a parcelas de restauración ecológica. Bol. Soc. Argent. Bot. 46 (Supl.): 77.
SOUZA, MDO C, A. M. BESERRA, D. C. MARTINS, V. V. REAL, R. A. SANTOS, V. S. RAO \& otros. 2009. In vitro and in vivo anti-Helicobacter pylori activity of Calophyllum brasiliense Camb. J. Ethnopharmacol. 123: 452-8.

STATGRAPHICS. 2009. Centurion XV. Statpoint Technologies, Inc. Warrenton VA, U.S.A.

TALAVERA STÉFANI, L. N., C. B. PERCUOCO, L. G. GIMÉNEZ, M. E. RODRÍGUEZ, A. E. CARDOZO, N. L. GONZÁLEZ \& otros. 2012. Obtención de microsatélites cloroplásticos en poblaciones argentinas de Calophyllum brasiliense. BAG. 23 (Supl.): 140.

VÁSQUEZ, W., K. A. THOMSEN \& D. JOKER. 2004. Desiccation and storage of seeds of Astronium graveolens and Calophyllum brasiliense, two native species of Costa Rica, pp. 278-294. International Plant Genetic Resources Institute. Forest And Landscape, Rome.

VÁSQUEZ-YANES, C., A. BATIS MUÑOZ, M. ALCOCER SILVA, M. GUAL DÍAZ \& C. SANCHEZ DIRSO. 1999. Árboles y arbustos potencialmente valiosos para la restauración ecológica y la reforestación. Reporte Técnico del Proyecto J 084, pp.100-102. CONABIO. Instituto de Ecología UNAM.

VERJÁN, J. C. GÓMEZ, R. R. CHILPA, M. A. CERBÓN CERVANTES, I. GONZÁLEZ SÁNCHEZ \& otros. 2013. Actividades citotóxica y genótoxica en células k562 de mammea a/ ba + a/bb provenientes de los extractos de Calophyllum brasilense. XXII Congreso de la Sociedad Ítalo Latinoamericana de Etnomedicina "Hernán Arguedas Soto". Puntarenas. p. 285 .

Recibido el 12 de noviembre de 2014, aceptado el 18 de diciembre de 2014. 